Fecha de recepción: diciembre 2020 Fecha de aprobación: febrero 2021 Fecha publicación: marzo 2021

\section{Islas de diseño: 3Re, una versión de sostenibilidad aplicada a pequeñas y medianas empresas fabricantes de calzado en la provincia de Tungurahua - Ecuador}

Edisson Viera Alulema ${ }^{(1)}$

\begin{abstract}
Resumen: En esta época de crisis e incertidumbre el Diseño debe plantear en presente y futuro un pensamiento de reflexión desde su accionar intermediario entre las perspectivas de ciencia, tecnología, economía, producción y ecología. Los nuevos escenarios del crecimiento global plantean un marco de estructura cultural desde lo proyectual hacia el mundo real en la asociatividad objeto-usuario-naturaleza, esta perspectiva planifica el bienestar de la sociedad, bajo el enunciado sostenible de la Comisión Brundtland (1987). La satisfacción de necesidades se argumenta en los discursos económicos desde dos enfoques: desarrollo y sustentabilidad. La argumentación de la sustentabilidad tiene dos dudas razonables: un crecimiento antieconómico debido a un crecimiento infinito en un sistema finito, y por otro lado la desigualdad social en la distribución de riqueza. Esta propuesta de Islas de Diseño rompe rutinas hacia formas más colectivas desde la innovación social, sincronizando el interés individual, social y ambiental en las 3Re: Re-descubrir, Re-organizar, Re-ingeniarse.

Las Islas de Diseño establecen espacios de certidumbre mediante un modelo de diseño sostenible para mejorar calidad de vida desde la gestión de la innovación social en los sistemas de producción a través de la solución de problemas y la creación de sentido, hacia una ecología de relaciones entre personas y su entorno.

A partir de este contexto proponemos las 3Re como enfoque en las pequeñas y medianas empresas manufactureras de calzado en la provincia de Tungurahua en la que se manufactura el $70 \%$ del calzado ecuatoriano y representa una producción de alrededor de 19 millones de pares de zapatos en 2016.
\end{abstract}

Palabras clave: diseño sostenible - desarrollo sostenible - sostenibilidad - economía circular - economía creativa.

[Resúmenes en inglés y portugués en las páginas 51-52]

(1) Universidad Técnica de Amabato (Ecuador). 


\section{Introducción}

En este contexto socioeconómico, la globalización ha ido superando períodos turbulentos llenos de dudas e incertidumbre, producto del constante cambio tecnológico. En este amplio y diverso horizonte contemporáneo, se estructura un mapeo real y virtual, repleto de datos de diversos campos en los que el diseño representa una disciplina coyuntural en los procesos productivos. En esta época, el desarrollo se ha basado en el crecimiento productivo, la industrialización y la mejora de la calidad de vida a través de la democratización del consumo (Bonsiepe, 1999).

El diseño adquiere una función esencial en tiempos de crisis, ya que integra la ciencia y la tecnología en la elaboración de objetos de la vida cotidiana considerando la asociación objeto-usuario. Con esta perspectiva, el modelo de desarrollo planifica el bienestar de la sociedad. Las declaraciones de la misma son principalmente sobre Desarrollo Sostenible originadas en la Comisión Brundtland: "Una que satisfaga las necesidades de la generación actual sin comprometer la capacidad de las generaciones futuras para satisfacer las suyas propias" (Brundtland, 1987).

La satisfacción de las necesidades de las generaciones transformadas de los consumidores se argumenta en los discursos económicos desde dos enfoques: de un lado el desarrollo y, para complementar, la sostenibilidad. Estas dos definiciones son difíciles de conciliar debido a su ambivalencia y complementariedad. Por sostenibilidad entendemos que la utilidad debe mantenerse en el tiempo y que no debe disminuir para las generaciones futuras. El desarrollo se entiende como el crecimiento de la sociedad teniendo en cuenta la integración económica en el proceso de globalización a través del libre mercado y la movilidad de capitales. Por otro lado, Canale, (2009) sostiene que la posibilidad de sostenibilidad es indefendible porque no se puede sostener desde el lado económico.

La argumentación de la sostenibilidad en el crecimiento global según Herman Daly, tiene dos dudas razonables. El primero, los límites ecológicos transforman el crecimiento económico en crecimiento anti-económico porque la macroeconomía tiene que considerar un ecosistema finito. En segundo lugar, la existencia de desigualdad social en la distribución de la riqueza, es decir, que el crecimiento no tiene que medirse solo con el valor del PIB (Daly, 2008), además, el contexto social desde la macroeconomía tiene varias consideraciones: 1) la única opción mejorar la calidad de vida es exportar a países ricos en mejores condiciones; 2) el crecimiento excesivo genera el aumento de brechas en los umbrales de riqueza entre países / personas / sociedades; 3) una mayor riqueza implica más contaminación en los productos y menos recursos naturales en los insumos de los procesos productivos; 4) los costos a considerar en esta teoría económica están relacionados con los recursos naturales y la contaminación.

En un mundo globalizado y económicamente integrado, con libre comercio y movilidad de capitales, la rentabilidad se transforma en el principal indicador de las exportaciones. La utilidad, producto de los procesos de fabricación de bienes y servicios exportados y utilizados en el comercio interno, cubren necesidades sociales y deben ser analizados a través de filtros de economía, producción y sociedad de acuerdo con la Figura 1. 


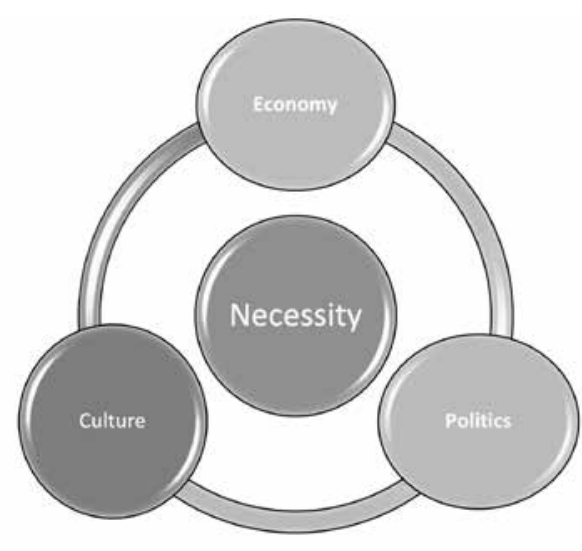

Figure 1.

Identification of necessities cycle.

La necesidad personal o colectiva identificada en el consumo proviene de la visión de la necesidad cultural en la apropiación de objetos forzados a una identidad en duda (Papanek, 2014). El hombre, desde el inicio de su existencia ha intentado cambiar su entorno con dispositivos que terminan siendo una extensión del cuerpo humano.

La pregunta inicial de la investigación sería: ¿Se están transformando objetivamente las necesidades en productos o servicios? Y si lo son, una segunda pregunta tiene que ser: ¿Cuál es el enfoque de desarrollo con el que se aborda la fabricación e industrialización del producto? En un sistema inestable de oferta y demanda debido a la rápida innovación como producto de la tecnología, prevalecen condiciones de mercado relacionadas con segmentaciones que, en general, tienen una falta de estructura de la investigación con objetivos poco claros y mala metodología. En el caso del diseño, es más un producto de las creencias del diseñador que una metodología disciplinaria real.

Se presentan dos enfoques económicos: por un lado, desde la economía lineal un ideal de usar-tirar que considera en el pasado, presente y futuro un desarrollo desequilibrado y un pensamiento de crecimiento desorganizado en un ecosistema que se consideraba infinito (Figura 2).

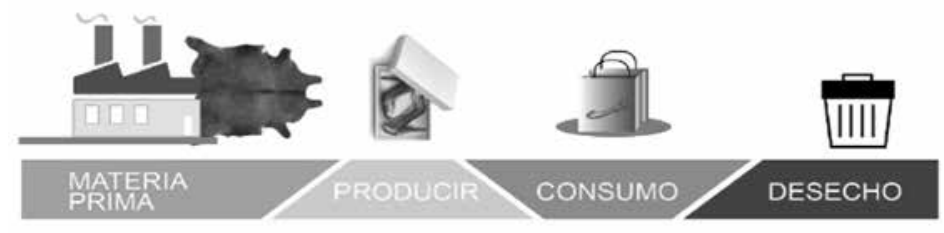

ECONOMÍA LINEAL
Figure 2. Lineal economy. 
Complementariamente, la propuesta de economía circular tiene como objetivo producir (respeto social) - reciclaje (respeto a la naturaleza), teniendo en cuenta las necesidades de las generaciones futuras. En este contexto, el objeto de análisis es el calzado diseñado en la provincia de Tungurahua en CALTU (Cámara del Calzado de Tungurahua).

El trabajo de investigación estimará el aporte del diseño sustentable en la economía de Tungurahua, en la que este último jugará un papel importante como uno de los factores de desarrollo junto a la tecnología como aliados en la creación de políticas que promuevan la creación de riqueza y empleo, de origen individual y competiciones grupales.

\section{Análisis del sector del calzado en la provincia de Tungurahua - Ecuador, visión de $3 \operatorname{Re}$}

Los enfoques analizados, en primer lugar, abordan el sector del calzado como símbolo de la industrialización de la moda en el proceso de globalización-consumo y cómo afrontan el contexto las pymes fabricantes de calzado en la provincia de Tungurahua. El segundo enfoque proviene del análisis del desarrollo sostenible en el modelo de Design Islands como versión de las 3Res de sostenibilidad.

Las Islas del Diseño son un cambio en la percepción de las nuevas sociedades a partir de una ecología de las relaciones entre las personas y las personas con su entorno. El ecosistema propuesto tiene que sintonizar sistemas productivos basados en una economía social, mediante la aplicación del 3Re: Redescubrir: trabajo colaborativo. Reorganizar: organización colaborativa productor / usuario. Rediseño: soluciones integrales.

En este contexto, definimos al diseño como un agente experto en la transformación de la linealidad a lo circular, creando nuevas formas de trabajo que conduzcan a la actividad productiva. De la dimensión local a la global, en la rutina de un mundo conectado. Estas nuevas formas de vida complementan los intereses individuales, sociales y ambientales hacia la sostenibilidad. El diseño como capacidad humana general incorporada en la solución de problemas es, además, la transición a una estructura de nuevos límites del planeta, formas de comunicación, reducción de brechas y mejora de los usos de la conectividad (Manzini, 2015).

En el Foro Social de Porto Alegre 2011 se proclamó “Otro mundo es posible”. La declaración del escritor indio Ahrundhari Roy en el mismo foro es el núcleo de esta investigación cuando afirma "Otro mundo no solo es posible, sino que está en camino [...]. Si presto atención, puedo escuchar cómo respira”. La idea de un mundo nuevo (Design Islands) será el motor de transformación, siempre que funcione como lucha por un mundo sostenible, a través de un diseño reflexivo y una cultura del diseño que se adapte a la innovación tecnológica y social (Manzini, 2015).

La economía ecuatoriana se ha caracterizado históricamente por producir y comercializar bienes primarios como estrategia de desarrollo socio-económico-industrial. Este modelo ha demostrado ser insostenible a medio y largo plazo, ya que no responde de forma eficaz en el contexto global. La presencia de un sistema competitivo, fuerte y desigual desde la hegemonía norte-sur en los mercados internacionales, favorece un nuevo modelo basado 
en el desarrollo de capacidades endógenas científico-tecnológicas, que revitalizan la innovación y el emprendimiento como objetivo táctico para fortalecer el sector productivo. El desarrollo mundial construido desde una perspectiva colonialista ha llevado a una crisis global que demuestra la imposibilidad de apoyar la extracción. Ese desarrollo formula relaciones de poder y comercio desigual. En este marco referencial de ideas dominantes, el progreso y el desarrollo invisibilizan la experiencia histórica de los pueblos que son la esencia fundamental de la sociedad (SENPLADES, 2009).

El patrón administrativo y demográfico en Ecuador determina que alrededor del $90 \%$ de las empresas se ubican en el sector urbano de las capitales de provincia. Asimismo, la escasa diversificación y el proteccionismo histórico del gobierno se establecen como una característica empresarial, lo que hace que los sectores industriales no sean competitivos (Cebrián, 1993).

El escenario económico del sector industrial del calzado según la organización mundial Worldfootwear, determina que el promedio de utilización por persona es de unos tres pares de zapatos al año, la producción mundial es de 23 billones de pares al año de los cuales el $57 \%$ se producen en China, lo que lo convierte en el país con mayor fabricación (Ramrattan et al., 2018).

Sobre Ecuador, Worldfootwear ubica al país en el puesto 28 en producción, con 40 millones de pares de zapatos por año. En el ranking de consumo ocupa el puesto 61 con 43 millones de pares al año. En el caso de la exportación, estamos en el lugar 50 con 6 millones de pares (21 millones de USD) enviados a Colombia (63\%) y Perú (33\%) .Las importaciones nos ubican en el lugar 102 con 9 millones de pares de zapatos (63 millones de USD). Los principales países de los que importa Ecuador son China y Vietnam.

Según CALTU, en la provincia de Tungurahua se produce el $65 \%$ del total nacional, con 18,6 millones de pares de zapatos. Se puede evidenciar, según un informe, que existe un fácil acceso a materia prima de alta calidad con una aceptación del $74 \%$, y maquinaria calificada con un 71,5\%. Entre los años 2016 y 2017, las importaciones de calzado aumentaron a 3 millones de pares, lo que representa un Incremento del 35\% La principal consecuencia social de este informe radica en la disminución de la fuerza laboral directa que representa un problema real de equidad económica y social, definido en una disminución de 400 empleos en el período de 2017 a 2018.

Sin embargo, a pesar de los datos estadísticos que reflejan un escenario con indicadores en mercado y materia prima y tecnología relativamente competitivos, existe desacuerdo en el sector industrial con la productividad, reflejada en sus limitados niveles de producción y exportación de producto, transformándolo en un sector que Depende de las medidas de protección del Estado ecuatoriano para sobrevivir en el mercado nacional y apenas existen en el mercado global. 


\section{Propuesta: visión sostenible 3Re "Centro de Innovación Tecnológica aplicada al calzado CITCA"}

El modelo presentado es parte del trabajo de investigación realizado en la tesis doctoral para el programa en diseño de la Universidad de Palermo, en el escenario descrito, al que aplicaremos la visión de sustentabilidad desde la 3Re de las Islas del Diseño desde el enfoque de economía circular mostrado en la Figura 3.

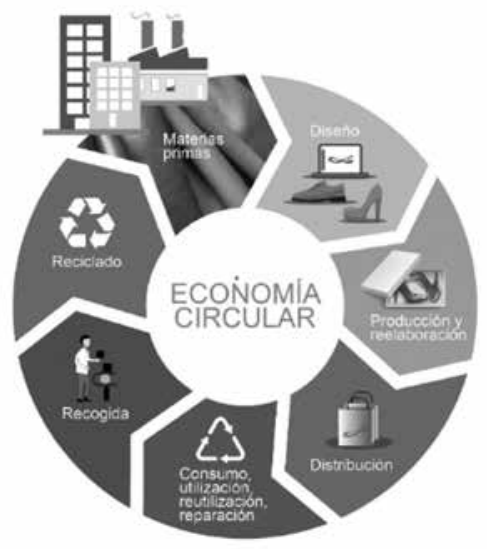

Figura 3. Economía circular.

1. Definir la necesidad: refiriéndose a los datos estadísticos, se podría plantear la siguiente pregunta: ¿Es posible mejorar la eficiencia productiva del sector del calzado en la provincia de Tungurahua? Con la información recopilada y la estructura conceptual definimos la necesidad de optimizar la productividad del sector del calzado de la provincia de Tungurahua según la Figura 4.

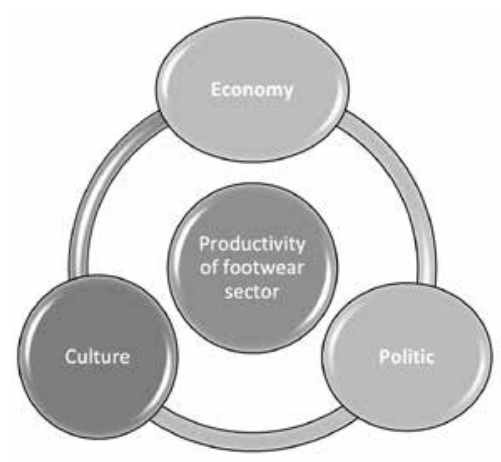

Figure 4.

Identificationof necessities CITCA. 
2. Redescubrir: el trabajo colaborativo tiene que proponerse desde varias perspectivas, en este caso, los actores para optimizar el sistema productivo del calzado se alinean en torno al desarrollo local: Municipio de Ambato, Cámara del Calzado de Tungurahua (CALTU), Técnico Universidad de Ambato.

La Universidad Técnica de Ambato, institución de educación superior con 51 años de existencia y 33 carreras, incluyendo diseño textil y de vestuario; 26 Posgrados que incluyen el Máster en Diseño de Calzado aprobado en 2020. Entre sus mecanismos para atender las necesidades de la sociedad, se encuentran 4 dominios. En este caso utilizaremos el segundo dominio de acuerdo con los planes estratégicos de desarrollo nacional y la adecuación de la economía circular: "Optimización de sistemas productivos, diseño y desarrollo urbanístico; contribuye al progreso tecnológico de la industria en sectores productivos y estratégicos de la región como el cuero y calzado, textiles y confecciones, construcción y metalmecánica, contribuyendo así al mejoramiento de las condiciones de vida, transporte y servicios básicos”.

El proyecto CITCA nace del fortalecimiento de la investigación en diseño, materiales y producción con el fin de fortalecer a las pequeñas y medianas empresas del sector. Los objetivos son encontrar soluciones a problemas del entorno de la empresa en modelos de gestión, procesos productivos e incorporación del diseño en cadenas de valor. En el marco del convenio UTA - CALTU se han realizado dos versiones del Diplomado en Tecnologías y Fabricación del Calzado.

Debido a la falta de desarrollo y consumo del diseño de calzado, CITCA surge como una necesidad de la propuesta del ciclo de identificación de necesidades, para beneficiar a las pequeñas y medianas empresas asociadas a CALTU, enfocado en 4 áreas:

1. Diseño para la creatividad y desarrollo del Observatorio de tendencias en calzado.

2. Modelado para buscar comodidad y calidad de calzado.

3. Producción eficiente y optimizada, hacia un consumo sostenible.

4. Calidad desde una visión global de las cadenas de valor.

El objetivo de CITCA es establecerse como un centro de apoyo al sector del calzado, proponer modelos y herramientas para la evaluación y validación de procesos productivos y creativos, garantizando la calidad de los productos derivados; Se propone una metodología Redescubrir y Reorganizar enfocada a la búsqueda de información sobre el entorno del sector del calzado para tener una percepción de sus necesidades y problemas. En la investigación se aplicarán métodos y técnicas cualitativas y cuantitativas, así como estudios de casos y el método Delphi, para establecer pautas y validez de un modelo de intervención de apoyo al calzado y su metodología de aplicación.

El centro brindará servicios especializados relacionados con los procesos de diseño, modelado, calidad y producción, mediante la implementación de talleres tecnológicos de vanguardia para ofrecer productos y servicios de calidad. Complementariamente, es fundamental la creación de un modelo de intervención de apoyo a las MIPYMES, con un enfoque especializado en el sector del calzado desde la ingeniería, el diseño y la economía. El 
más importante es el fortalecimiento de la calidad del producto y la apertura de mercados a través de la intervención del CITCA.

Beneficiará a 75 empresas asociadas en CALTU, 40 que pertenecen a la provincia de Tungurahua, incluida la asociación de curtidores como parte de la cadena de valor. Otro grupo importante de apoyo son los aproximadamente 300 estudiantes promedio por ciclo académico de la Carrera de Diseño Textil y de Confección, FDA - UTA, y la oportunidad para 23 profesores de investigación FDA - UTA, así como investigadores de otras carreras de UTA, universidades e instituciones de la zona 3 que estén interesados en la generación de proyectos locales y regionales.

El proyecto CITCA transferirá resultados a través de publicaciones científicas, publicaciones técnicas, desarrollo de Congresos de Diseño (IV Congreso de Investigación en Diseño y II Congreso de Diseño de Calzado 2020), Modelos de intervención aplicados a empresas del sector de la piel y el calzado.

En cuanto a los aspectos bioéticos que afectan directamente al ecosistema, especialmente al ser humano a través de la contaminación de sus recursos hídricos, se propondrán alternativas de procesos y materiales amigables que brinden una conciencia de consumo y producción; mediante la investigación en el ciclo de vida del producto y la transferencia de conocimiento y tecnología en un mercado eco-sostenible.

\section{Referencias}

Bonsiepe, G. (1999). Del Objeto a la interfase (Ediciones).

Brundtland, G. H. (1987). Informe de la Comisión Mundial sobre Medio Ambiente y el Desarrollo: Nuestro futuro común. Documentos de Las Naciones, Recolección de Un ..., 416. http://scholar.google.com/scholar?hl=en\&btnG=Search\&q=intitle:Informe+de+la + comision + mundial+sobre $+\mathrm{el}+$ medio + ambiente $+\mathrm{y}+\mathrm{el}+$ desarrollo.+nuestro+futuro + comun\#5

Canale, G. (2009). Económica Social Ambiental. 1-21.

Cebrián, F. (1993). Anotaciones a La Estructura Espacial De La Industria En Ecuador. Papeles de Geografia, 19, 103-114.

Devalle, V. (2009). La Travesía de la Forma: emergencia y consolidación del Diseño Gráfico (1a ed.). Paidos.

Daly, H. E. (2008). Aportes.

Fonseca Reis, A. C. (2018). Economía creativa. In Itaú Cultural.

Manzini, E. (2015). Cuando todos diseñan: Una introducción al diseño para la innovación social (1a ed.).

Manzzini, E. (2016). Design Culture and Dialogic Design. Design Issues, 32, 52-59. https:// doi.org/10.1162/DESI/_a_00364

Papanek, V. (2014). Diseñar para el mundo real (segunda). El Tinter.

Ramrattan, L.; Szenberg, M.; Ramrattan, L. \& Szenberg, M. (2018). The Footwear Industry. Distressed Us Industries in the Era of Globalization, 37-52. https://doi. org/10.4324/9781351159807-3 
SENPLADES. (2009). Plan Nacional para el Buen Vivir 2009-2013: Construyendo un Estado Plurinacional e Intercultural.

\begin{abstract}
In this age of crisis and uncertainty, the design has to propose, in present and future, a reflection thinking in the perspectives of science, technology, economy, production, and ecology. The new scenarios of global growth pose a cultural structure from the project to the real world by associating object-user-nature. This plans society wellness under the statement of Brundtland Commission (1987).

The satisfaction of necessities is argued in the economic speeches from two approaches: development and sustainability. The argumentation of the last has two reasonable doubts: an antieconomic growth due to an infinite growth in a finite system, and on the other hand the social inequality in wealth distribution. This proposal of Design Islands breaks routines towards more collective ways, from social innovation syncing individual, social, and environmental interests in 3Re: Re-discover, Re-organize, and Re-engineer.

The Design Islands establish certainty via a sustainable design model to improve the quality of life from managing social innovation in production systems, solving problems and creating meaning towards an ecology of relationships between people and their environment. From this context, we propose the $3 \mathrm{Re}$ as an approach for small and medium footwear manufacture companies in Tungurahua province in which $70 \%$ of Ecuadorian footwear is manufactured, with a production of around 19 million of pairs of shoes in 2016.
\end{abstract}

Keywords: sustainable design - sustainable development - sustainability - circular economy - creative economy.

Resumo: O centro prestará serviços especializados relacionados a processos de design, modelagem, qualidade e produção, por meio da implantação de oficinas tecnológicas de ponta para oferecer produtos e serviços de qualidade. Complementarmente, é imprescindível a criação de um modelo de intervenção de apoio às MPME, com enfoque especializado no setor do calçado desde a engenharia, design e economia. O mais importante é o reforço da qualidade dos produtos e a abertura dos mercados através da intervenção da CITCA.

Beneficiará 75 empresas associadas em CALTU, 40 pertencentes à província de Tungurahua, incluindo a associação do curtidor como parte da cadeia de valor. Outro importante grupo de apoio são os cerca de 300 alunos médios por ciclo acadêmico da Carreira em Design Têxtil e de Confecção, FDA - UTA, e a oportunidade para 23 professores pesquisadores da FDA - UTA, além de pesquisadores de outras carreiras da UTA, universidades e instituições da zona 3 que têm interesse na geração de projetos locais e regionais.

O projeto CITCA irá repassar resultados através de publicações científicas, publicações técnicas, desenvolvimento de Congressos de Design (IV Congresso de Pesquisa em Design e II Congresso de Design de Calçados 2020), Modelos de intervenção aplicados a empresas do setor coureiro-calçadista. 
Em relação aos aspectos bioéticos que afetam diretamente o ecossistema, especialmente o ser humano pela contaminação de seus recursos hídricos, serão propostas alternativas de processos e materiais amigáveis que proporcionem uma consciência de consumo e produção; pela pesquisa do ciclo de vida do produto e transferência de conhecimento e tecnologia em um mercado eco-sustentável.

Palavras chave: design sustentável - desenvolvimento sustentável - sustentabilidade - economia circular - economia criativa.

[Las traducciones de los abstracts fueron supervisadas por el autor de cada artículo] 